\title{
Does diffusion-weighted magnetic resonance imaging help in the detection of renal parenchymal disease and staging/prognostication in chronic kidney disease?
}

\author{
Vijinder Arora ${ }^{C, D, E, F}$, Jasmin Khatana ${ }^{A, B, C, D, E, F}$, Kunwarpal Singh, ${ }^{D, E, F}$ \\ Shri Guru Ram Das Institute of Medical Sciences and Research, Punjab, India
}

\begin{abstract}
Purpose: Diffusion-weighted imaging (DWI) in renal diseases is an upcoming modality, and its utility as an additional marker is yet to be proven. This study was intended to find the relationship between apparent diffusion coefficient (ADC) values with renal function tests and stages of chronic kidney disease (CKD) to assess renal dysfunction, and to label a cut-off for normal renal function and dysfunction.

Material and methods: A prospective diagnostic study was conducted on 120 patients: 60 with deranged renal function tests (RFT) and 60 with normal RFT. DWI using a 1.5-Tesla MRI (at $b$-values of 0 and $500 \mathrm{~s} / \mathrm{mm}^{2}$ ) was done. A region of interest of size 1-2 $\mathrm{cm}^{2}$ was placed on renal parenchyma in the region of medulla, one each, over the superior, mid, and lower regions of each kidney separately. ADC values were recorded for renal parenchyma and compared.

Results: In patients with renal dysfunction ADC values were significantly lower than in patients with normal function $\left(1.75 \pm 0.25\right.$ vs. $2.28 \pm 0.21$ of right kidney and $1.79 \pm 0.17$ vs. $2.29 \pm 0.21$ of left kidney $\left.\left[\times 10^{-3} \mathrm{~mm}^{2} / \mathrm{s}\right] ; p=0.001\right)$ ADC values of different stages of CKD showed a decreasing trend with increasing stage.

Conclusions: ADC values taken at all poles to get focal involvement of the kidney can be used to measure each kidney separately, and values can be individually correlated with the elevated renal parameters. The cut-off value of the mean ADC for individual kidneys was $>2.28\left(\times 10^{-3} \mathrm{~mm}^{2} / \mathrm{s}\right)$ in normal renal function and $<2.00\left(\times 10^{-3} \mathrm{~mm}^{2} / \mathrm{s}\right)$ in renal dysfunction.
\end{abstract}

Key words: diffusion-weighted imaging, renal function tests, chronic kidney disease, apparent diffusion coefficient, renal parenchymal disease.

\section{Introduction}

Renal disease progression can be assessed by monitoring renal function. Periodic assessment of renal function is necessary for optimum management of a patient with suspected or proven renal disease. Serum creatinine (S Cr) and blood urea (BU) are useful for monitoring renal function; however, these indirect measures of renal filtration are imperfect and cannot assess single kidney function [1]. Elevated serum creatinine is often revealed only in the late stages of disease, when extensive and sometimes irreversible renal damage takes place [2]. The estimated glomerular filtration rate (eGFR) is considered the best indicator of renal function. It can also be detected by creatinine clearance [3]. Due to the limitations of serum markers, imaging techniques are gaining importance in the evaluation of renal function [4].

Ultrasonography (USG) and computed tomography (CT) scan provide suitable anatomic images but limited functional information. Although USG may show changes

Correspondence address:

Dr. Vijinder Arora, Shri Guru Ram Das Institute of Medical Sciences and Research, Shri Hargobindpur Rd, Vallah, Qila Jiwan Singh, Punjab 143501, India, e-mail: drvijinderarora338@gmail.com

Authors' contribution:

A Study design · B Data collection · C Statistical analysis · D Data interpretation · E Manuscript preparation · F Literature search · G Funds collection 
in renal echogenicity, it suffers from operator dependency and lacks neutrality. In CT scan there is exposure to ionizing radiation. In addition, it requires use of iodinated contrast material, which is unfavourable in patients with renal dysfunction [5].

Magnetic resonance imaging (MRI) can adequately study the morphology of kidneys without any radiation exposure or need for iodinated contrast. Diffusion-weighted magnetic resonance imaging (DWI) is an advanced MRI technique used to characterize tissues based on the Brownian motion of water molecules within them [1]. Diffusion-weighted imaging is designed to analyse random microscopic motion or diffusion of water in tissue and does not require exogenous contrast agents [2]. The apparent diffusion coefficient (ADC) is a quantitative parameter, which is calculated from the DWI that pools the properties of capillary perfusion and water diffusion. DWI in kidneys is useful in assessing the renal function because it has increased blood flow and regulates water fluid and electrolyte balance $[1,6]$. Recent studies have already shown the potential value of this method in the evaluation of various renal diseases, such as renal infection, renal ischaemia, pyonephrosis, and diffuse renal disease [5].

The application of ADC in renal dysfunction and fibrosis in which there is worsening renal function portrays a decrease in water reabsorption [2]. The aspects of the specific transport and urinary concentration mechanisms could have a considerable effect on the ADC values of various regions of the kidneys. Moreover, alterations in water mobility may be expected in various disease states of the kidneys [7].

This study was conducted to explore the relationship between ADC values of renal parenchyma, serum markers, and stages of chronic kidney disease (CKD). We also envisioned to determine cut-off ADC values to identify renal dysfunction.

\section{Material and methods}

A single institutional diagnostic study with a sample size of 120 patients (60 cases and 60 controls) was completed during 21 months at our institute. Informed consent was obtained. Patients of age group 11-80 years and of either gender were included. In the conducted study, 65 patients were female and 55 patients were male. Clinically, 68 patients were hypertensive, 51 were diabetic, and 34 presented with both hypertension and diabetes mellitus. Patients who had a cut-off value of $>1.3 \mathrm{mg} / \mathrm{dl}$ for S Cr were included in the study group. Patients were classified into CKD stages (G1-G5) based on the disease severity, as per the level of serum creatinine and parameters according to the UK Renal Association. G1 shows normal and high eGFR $>90 \mathrm{ml} / \mathrm{min} / 1.73 \mathrm{~m}^{2}$, G2 shows mild reduction related to normal range for young adult $60-89 \mathrm{ml} / \mathrm{min} / 1.73$ $\mathrm{m}^{2}$, G3a-b show mild-moderate and moderate-severe re- duction $30-59 \mathrm{ml} / \mathrm{min} / 1.73 \mathrm{~m}^{2}$, G4 shows severe reduction $15-29 \mathrm{ml} / \mathrm{min} / 1.73 \mathrm{~m}^{2}$, and G5 shows kidney failure $<15 \mathrm{ml} / \mathrm{min} / 1.73 \mathrm{~m}^{2}$ [8]. eGFR was calculated by using the Modification of Diet in Renal Disease Study (MDRD): $186 \times(\text { creatinine } / 88.4)^{-1.154} \times(\text { Age })^{-0.203} \times(0.742$ if female $)$ $\times$ (1.210 if black) [9].

All the patients were examined under a 1.5-Tesla MRI scanner (Philips Achieva D-stream) in a supine position after placing a body coil over the abdomen. DWI-MRI was done with TR $1600 \mathrm{~ms}$, TE $70 \mathrm{~ms}$, EPI factor 95, FOV $249 \times 380 \mathrm{~mm}^{2}$, and distance factor $30 \%$. Respiratory triggered FS (spectral fat suppression) spin echo-echo planar imaging (SE-EPI) axial diffusion-weighted sequence at $b$-value 0 and $500 \mathrm{~s} / \mathrm{mm}^{2}$ was conducted. Other parameters were flip angle $90^{\circ}$, slice thickness $4 \mathrm{~mm}$, number of averages 2, receiver bandwidth $1735 \mathrm{H}_{3}$ /pixel, and acquisition time 4-5 min. DW sequence was respiratory triggered using the navigator-trigger prospective acquisition correction technique (PACE), and the position of the diaphragm was assessed periodically by the navigator echoes. Apparent diffusion coefficient (ADC) maps were extracted automatically on a voxel-by-voxel basis.

$\mathrm{ADC}$ values were measured quantitatively by assigning a region of interest of size $1-2 \mathrm{~cm}^{2}$. The region of interest (ROI) was placed on the renal parenchyma in the region of the medulla. ADC values were not separately measured for the renal cortex because it is hard to place the ROI cursor precisely in the region of the cortex, especially in patients with severely contracted kidneys. ROIs were placed, one each, over the superior, mid, and lower regions of each kidney separately. The mean ADC of these 3 values was analysed for each kidney separately. The apparent diffusion coefficient was measured as mean \pm standard deviation $\left(\mathrm{A} \times 10^{-3} \mathrm{~mm}^{2} / \mathrm{s}\right)$.

The collected data was analysed with SPSS 23.0 version. To describe data, descriptive statistics like frequency analysis and percentage analysis were used for categorical variables, and for continuous variables the mean and standard deviation (SD) were used. Independent $t$-test was used to find significant differences between the bivariate samples in independent groups (normal and abnormal). One-way ANOVA with Tukey's post-hoc test was used for multivariate scrutiny. To assess the correlation between the variables Pearson's correlation was used. Receiver operating characteristic curves (ROC) were drawn to calculate the area under the curve (AUC) to distinguish the 2 groups, and cut-off ADC values were calculated to achieve the highest average sensitivity and specificity. The $\chi^{2}$ test was used to find connotation in categorical data. A probability value of 0.05 was considered as a noteworthy level in all the above statistical tools used.

\section{Results}

The study group comprised 120 patients, which included 60 patients with increased S Cr with reduced eGFR and 
60 patients with normal S Cr and eGFR. Out of the 60 patients with increased $\mathrm{S} \mathrm{Cr}, 47$ patients were said to have $\mathrm{CKD}$, and the remaining 13 patients had deranged $\mathrm{S} \mathrm{Cr}$ levels but normal eGFR levels. According to the UK Renal Association, CKD was classified using the MDRD method, and the eGFR was calculated. Appropriately, CKD is labelled when eGFR is lower than $<60 \mathrm{ml} / \mathrm{min} / 1.73 \mathrm{~m}^{2}$, therefore it should be diagnosed from stage 3 onwards. Out of the 60 patients with deranged S Cr, 13 had normal eGFR, who were grouped in G1-G2 stage, 16 patients were grouped in G3a-b stage, 17 patients were grouped in G4 stage, and 14 patients were grouped in G5 stage. Mean creatinine level for patients with renal dysfunction was $3.19 \pm 4.00 \mathrm{mg} / \mathrm{dl}$ (range $0.09-23.01 \mathrm{mg} / \mathrm{dl}$ ) and mean blood urea $(\mathrm{BU})$ was $36.66 \pm 40.12 \mathrm{mg} / \mathrm{dl}$ (range $7.7 \pm$ $293.3 \mathrm{mg} / \mathrm{dl})$.

The mean ADC value of renal parenchyma in patients with renal dysfunction was significantly lower than in patients with normal renal function $(1.75 \pm 0.25$ vs. $2.28 \pm$ 0.21 of right kidney and $1.79 \pm 0.17$ vs. $2.29 \pm 0.21$ of left kidney $\left.\left(\times 10^{-3} \mathrm{~mm}^{2} / \mathrm{s}\right) ; p=0.001\right)$ (Figure 1$)$. For a cutoff ADC value of $2.00\left(\times 10^{-3} \mathrm{~mm}^{2} / \mathrm{s}\right)$, the sensitivity was $56 \%$, specificity was $91 \%$, and $95 \%$ confidence intervals (values below cut-off indicated renal dysfunction). In addition, ADC values higher than $2.28 \pm 0.19\left(\times 10^{-3} \mathrm{~mm}^{2} / \mathrm{s}\right)$ were seen only with normal renal function $(100 \%$ sensitivity). The positive predictive value was $87 \%$ and negative predictive value was $67 \%$.

There was a significant inverse correlation between $\mathrm{ADC}$ values of renal parenchyma and S Cr levels. The mean ADC value of renal parenchyma in patients with raised S Cr was significantly low $\left(1.95 \pm 0.27\left[\times 10^{-3} \mathrm{~mm}^{2} / \mathrm{s}\right]\right.$; $p=0.001$ ) (Table 1). Similarly, a significant inverse correlation was also observed between ADC values of renal parenchyma and BU levels $\left(1.96 \pm 0.27\left[\times 10^{-3} \mathrm{~mm}^{2} / \mathrm{s}\right]\right.$; $p=0.001$ ) (Table 2).

The mean ADC values of different stages of CKD were significantly different from each other $(p=0.001)$ and showed decreasing trend with increasing stage (Figure 2). Grade 1, 2, 3A-3B, 4, and 5 had average ADC values of
$2.32 \pm 0.22,2.26 \pm 0.26,2.08 \pm 0.30,1.94 \pm 0.21$, and 1.89 $\pm 0.25\left(\times 10^{-3} \mathrm{~mm}^{2} / \mathrm{s}\right)$, respectively (Figures $3 \mathrm{~A}-\mathrm{B}$ ).

\section{Discussion}

DWI can be used in the diagnosis of renal diseases because it is non-invasive and does not require a contrast agent. It can be included in a routine abdominal MRI protocol, which will not only help in the identification of morphological changes but also in evaluating functional changes in the kidney. The mean ADC value of renal parenchyma with abnormal renal function has been found to be significantly lower than in patients with normal renal function showing high S Cr levels [5].

Low ADC values in renal parenchymal disease can be explained by reduced perfusion as well as reduced water diffusion. In addition, DWI can also be influenced by water content of kidney and renal blood volume. Furthermore, evaluation of the intra-parenchymal physiological process such as blood flow, water content, and tubular flow may alter the ADC values [10]. The reason for reduction in glomerulosclerosis, tubular atrophy, and interstitial

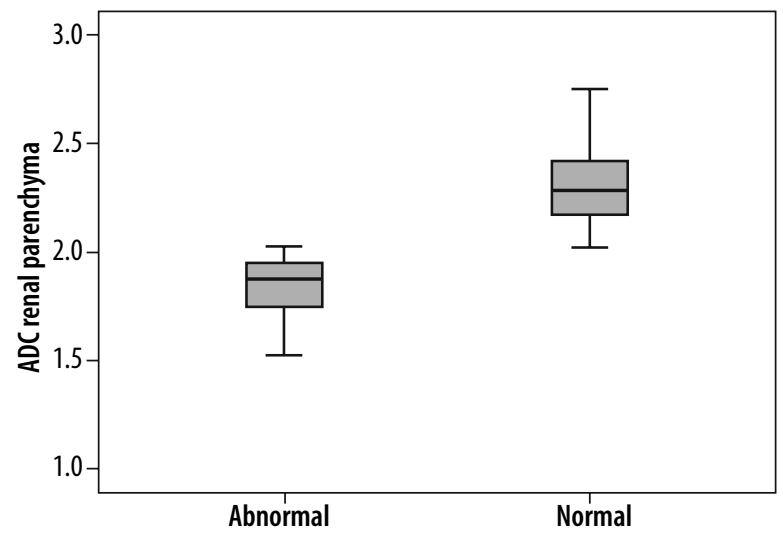

Figure 1. Box and whisker plot of renal parenchyma ADC in patients with renal dysfunction and those with normal renal function showing lower $A D C$ values associated with renal dysfunction. The lines within the box represent median values. The T bars that extend from the boxes (whiskers) are expected to include approximately $95 \%$ of the data (assuming normal distribution)

Table 1. Depicting correlation between $A D C$ of renal parenchyma and serum creatinine

\begin{tabular}{|l|c|c|c|c|c|}
\hline \multirow{2}{*}{ Serum creatinine } & \multicolumn{2}{|c|}{ ADC renal parenchyma } & \multicolumn{2}{c|}{$95 \% \mathrm{Cl}$} & $p$-value \\
& Mean & SD & Lower & Upper & \\
\hline Normal & 2.272 & 0.235 & 0.227 & 0.408 & 0.001 \\
\hline Abnormal & 1.956 & 0.274 & & & \\
\hline
\end{tabular}

Table 2. Depicting correlation between ADC of renal parenchyma and blood urea nitrogen

\begin{tabular}{|l|c|c|c|c|c|}
\hline \multirow{2}{*}{ Blood urea nitrogen } & \multicolumn{2}{|c|}{ ADC renal parenchyma } & \multicolumn{2}{c|}{$95 \% \mathrm{Cl}$} & \multicolumn{2}{c|}{$p$-value } \\
& Mean & SD & Lower & Upper & \\
\hline Normal & 2.277 & 0.238 & 0.217 & 0.404 & 0.001 \\
\hline Abnormal & 1.966 & 0.273 & & & \\
\hline
\end{tabular}




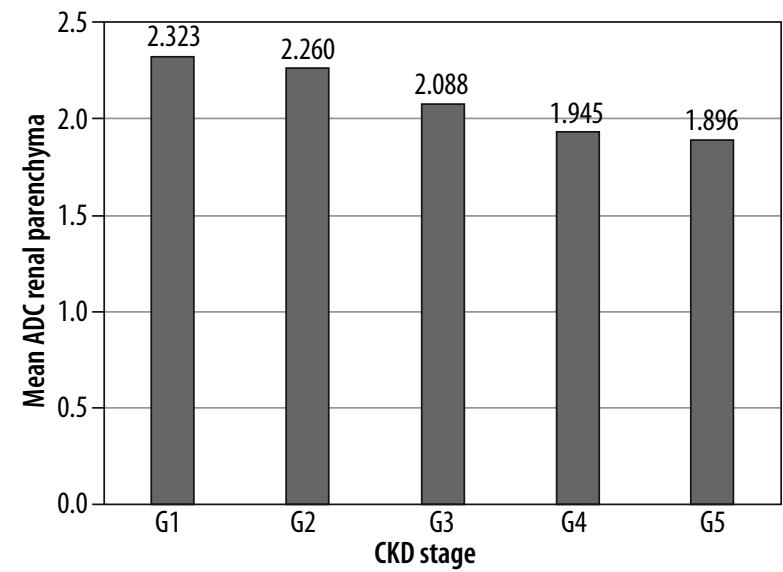

Figure 2. Cone graph of renal parenchyma ADC in concordance to chronic kidney disease

fibrosis can be attributed to the decrease of free movement of water molecules in intracellular and extracellular space.

Some of the studies on ADC values of renal parenchyma separated ADC values into the cortex and medulla, whereas others provided a mean ADC value for the entire parenchyma [11]. We placed ROIs in the medullary region, considering the fact that fibrosis occurs in dysfunctional kidneys and measurement at the cortex becomes impossible.

In our study, the mean $\mathrm{ADC}$ value of renal parenchyma in patients with renal dysfunction was significantly lower than in patients with normal renal function. Ichikawa et al. [3] stated that the ADC value decreases as renal function decreases. Low ADC values in renal parenchymal disease can be attributed to reduced perfusion as well as reduced water diffusion. Fukuda et al. [7] also reported that in either portion of the kidney at any $b$-value range, the ADCs for the kidneys of patients with high serum creatinine levels were lower than those of patients with a normal serum creatinine level.

The ADC value of renal parenchyma decreases with increasing S Cr and BU levels. Goyal et al. [4] conducted a study on 88 patients of whom 22 had renal dysfunction, and 15 of these were known cases of CKD. They noted a significant inverse correlation between ADC values of re- nal parenchyma and S Cr/BU levels $(R=-0.530$ and -0.502 , respectively). A statistically negative correlation was also found in a study done by Kadihan et al. [6], who evaluated 110 patients by imaging the upper abdomen for different reasons. They concluded statistically significantly higher ADC values in stage 3 , stage 4 , and stage 5 of CKD with negative correlation of $31.6 \%$ between serum creatinine values and $\mathrm{ADC}$ values. Similarly, we also noted a negative correlation between $\mathrm{ADC}$ values and $\mathrm{S} \mathrm{Cr} / \mathrm{BU}$ levels.

In the present study, ADC values higher than 2.28 $\left(\times 10^{-3} \mathrm{~mm}^{2} / \mathrm{s}\right)$ were found in patients with normal renal function. This was in concordance with Lavdas et al. [12], who proposed $\mathrm{ADC}$ values for normal renal parenchyma ranging between 2.25 and $2.75\left(\times 10^{-3} \mathrm{~mm}^{2} / \mathrm{s}\right)$ in both males and females. Similar cut-off ADC values for normal renal function were given by Ries et al. [13], Theony et al. [14], and Nanimoto et al. [15] at $2.30 \pm 0.24,2.17 \pm 0.12$, and $2.84 \pm 0.72\left(\times 10^{-3} \mathrm{~mm}^{2} / \mathrm{s}\right)$, respectively.

Eldin et al. [16] reported a comparable average ADC value of the kidneys in cases at $1.85 \times 10^{-3} \pm 0.24 \mathrm{~mm}^{2} / \mathrm{s}$, with a minimum of 1.20 and a maximum of 2.30 . Likewise, in our study the average ADC value was $2.00 \times$ $10^{-3} \mathrm{~mm}^{2} / \mathrm{s}$, with a minimum of 1.75 and maximum of 2.28. Sivakumar et al. [1], noticed low ADC value of $<1.986 \times 10^{-3} \mathrm{~mm}^{2} / \mathrm{s}$ on the right side and 1.970 $\times 10^{-3} \mathrm{~mm}^{2} / \mathrm{s}$ on the left side with raised creatinine level, which was significant at $0.001(<0.05)$ on both sides. Similarly, in our study low ADC values of $1.750 \times 10^{-3}$ $\mathrm{mm}^{2} / \mathrm{s}$ and $1.798 \times 10^{-3} \mathrm{~mm}^{2} / \mathrm{s}$ were seen on the right and left side, respectively.

A meta-analysis was conducted by Haitan et al. [17], which showed that DWI is a useful imaging method to evaluate renal function and that it can distinguish early stages of CKD from normal kidneys and aid in the staging of CKD. A significant linear correlation was found between renal parenchymal ADC values and eGFR in CKD patients. As the eGFR decreased so did the parenchymal $\mathrm{ADC}$ value, especially in CKD patients. This is in concordance with the results of $\mathrm{Xu}$ et al. [18]. Low ADC values are statistically meaningful with increasing stage of chronic kidney disease. Toya et al. [19] had similar cut-off GFR

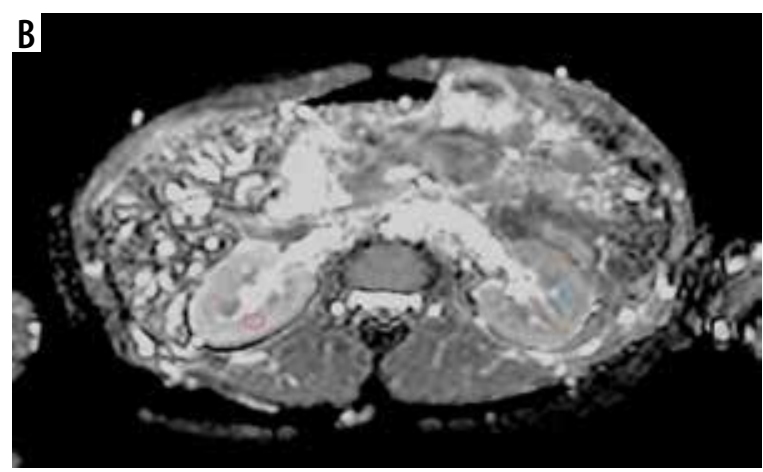

Figure 3. ADC map (derived from DW-MRI) (A) in a chronic kidney disease (CKD; stage 5) patient showing restricted diffusion in the renal parenchyma bilaterally with a mean $A D C$ value of $1.53 \times 10^{-3} \mathrm{~mm}^{2} / \mathrm{s}$. $A D C$ map (B) in a patient with normal renal function showing no restriction of diffusion and mean $A D C$ value was $2.27 \times 10^{-3} \mathrm{~mm}^{2} / \mathrm{s}$. The circles depict example of ROI placement 
values; they found a significant difference between stage 4 and 5 but not between stage 3 and 4 disease. Also, in our study it was noted that diabetic patients showed lower ADC values in comparison to healthy subjects. Quantitative kidney DWI may be useful in the follow-up of CKD patients in serial studies and in predicting the progression or regression of the kidney disease [20].

There were a few exceptions noted in the present study, in which the S Cr levels were increased but eGFR values were within the normal range. Some patients presented with conditions such as dehydration due to gastroenteritis, trauma post road traffic accident, intake of certain dietary supplements, and drug therapy such as ace inhibitors, diuretics, and chemotherapy agents. Therefore, not all patients presenting with raised S Cr levels were identified as having CKD.

Various studies have been conducted using different $b$-values. DWI was performed by Ichikawa et al. [3], using a range of low $\left(<50 \mathrm{~s} / \mathrm{mm}^{2}\right)$ and high $\left(>200 \mathrm{~s} / \mathrm{mm}^{2}\right)$ $b$-values to measure diffusion and perfusion separately. In our protocol we set $b$-values at $\left(0\right.$ and $\left.500 \mathrm{~s} / \mathrm{mm}^{2}\right) . \mathrm{Xu}$ et al. [18] reported significantly lower ADCs in impaired than in normal kidneys and observed a positive correlation between the ADCs and GFR values using $b$-values of 0 and $500 \mathrm{~s} / \mathrm{mm}^{2}$. According to Xue et al. [21], to get more precise results, $3 b$-values are preferred over $2 b$-values, which balances the T2 shine through and capillary effect, resulting in true diffusion and not individual effects of diffusion and perfusion. Chandrana et al. [22] suggested using 2 diffusion encoding strengths of $b$-values of 0 and 500 to give a decay constant, in accordance with our study.

We found the sensitivity of DWI to be $56 \%$ and specificity $91 \%$. Goyal et al. [4] reported sensitivity of $58.8 \%$ and specificity of $79.4 \%$. Considering the sensitivity for true positive dysfunction, the accuracy is variable depending on the duration of related symptoms, whether acute or chronic.

$\mathrm{Xu}$ et al. [23] found a negative correlation between ADC values and aging. Aging kidney is characterized by a decrease in renal blood flow and glomerular filtration rate. In our study, also, it was noted that in a few of the elderly controls ADC values were low even with a normal S Cr level.

There are a few limitations of our study. First, the sample size of the patients was small. A greater number of patients is required to substantiate the results. Some of the patients in the study were not primarily enrolled for evaluation of renal function only. Patients coming for evaluation of focal renal lesions and/or any other abdominal condition with increased serum creatinine were also enrolled. Additionally, we could not categorize the patients with low ADC values in our study into acute or chronic renal disease.

\section{Conclusions}

DWI is emerging as a promising tool to assess renal function. DWI as a guide to visualizing renal function demonstrates advantages of short time of acquisition, non-invasive nature, and no exposure to ionizing radiation/contrast material. The ADC value can be implemented as an additional marker for renal dysfunction. It can also be helpful in staging and deciding prognosis of CKD patients. Quantitatively renal DWI plays a role in the staging of CKD patients and making clinical decisions in follow-up of such patients to monitor the progression of disease. ADC values of individual kidney can be assessed separately, values can be taken at all poles to get a focal involvement of the kidney and can be individually correlated with the elevated renal parameters. Hence, DWI also shows the potential to locate the site for performing renal biopsy. A cut-off value of mean $\mathrm{ADC}$ for individual kidney $>2.28\left(\times 10^{-3} \mathrm{~mm}^{2} / \mathrm{s}\right)$ can signify normal renal function and $<2.00\left(\times 10^{-3} \mathrm{~mm}^{2} / \mathrm{s}\right)$ can signify renal dysfunction. Its drawbacks include limited availability and high cost.

It must be emphasized that DWI is in no way a substitute for serum markers or renal scintigraphy for the assessment of renal dysfunction; rather it is an additional prompting tool.

\section{Conflicts of interest}

The authors report no conflict of interest.

\section{References}

1. Sivakumar K, Ramesh D, Kailasanathan N. Diffusion-weighted magnetic resonance imaging (DWIMRI) in renal diseases is an emergency field and its utility is yet to be fully realized. Stanley Medical Journal 2016; 3: 38-45.

2. Zhang J, Rusinek H, Chandarana $H$, et al. Functional MRI of the kidneys. J Magn Reson Imaging 2013; 37: 282-293.

3. Ichikawa S, Motosuji U, Ichikawa T, et al. Intravoxel incoherent motion imaging of the kidneys: alterations in diffusion and perfusion in patients with renal dysfunction. Magn Reson Imaging 2013; 31: 414-417.
4. Cova M, Squillaci E, Stacul F, et al. Diffusion weighted MRI in the evaluation of renal lesions: preliminary results. Br J Radiol 2004; 77: 851-857.

5. Goyal A, Sharma R, Bhalla A, et al. Diffusion weighted MRI in assessment of renal dysfunction. Indian J Radiol Imaging 2012; 22: 155-159.

6. Safak K, Ayyildiz M, Unel S, et al. The relationship of ADC values of renal parenchyma with CKD stage and serum creatinine levels. Eur J Radiol Open 2015; 3: 8-11.

7. Fukuda Y, Ohashi I, Hanafusa K, et al. Anisotropic diffusion in kidney: apparent diffusion coefficient measurements for clinical use. J Magn Reson Imaging 2000; 11: 156-160. 
8. The Renal Association Founded 1950. Ron Cullen. Renal Association 2020. Available from: https://renal.org/information-resources/theuk-eckd-guide/ckd-stages/.

9. UKidney Internet school of Nephrology. 2007-2020 BrightBean Solutions Inc. Available from: https://ukidney.com/nephrology-resources/egfr-calculator.

10. Prasad PV, Thacker J, Li LP, et al. Multi-parametric evaluation of chronic kidney disease by MRI: a preliminary cross-sectional study. PLoS One 2015; 10: e0139661.

11. Colakoglu H. Apparent diffusion coefficient values of renal parenchyma in healthy adults: a 3 Tesla MRI study. Eur J Ther 2019; 25: 64-68.

12. Lavdas I, Rockall A, Castelli F, et al. Apparent diffusion coefficient of normal abdominal organs and bone marrow from whole-body DWI at 1.5 T: the effect of sex and age. AJR Am J Roentgenol 2015; 205: 242-250.

13. Ries M, Jones RA, Bassesu F, et al. Diffusion tensor MRI of the human kidney. J Magn Reson Imaging 2001; 14: 42-49.

14. Thoeny HC, Zumstein D, Simon-Zoula S, et al. Functional evaluation of transplanted kidneys with diffusion-weighted and BOLD MR imaging: initial experience. Radiology 2006; 241: 812-821.

15. Namimoto T, Yamashita Y, Mitsuzaki K, et al. Measurement of the apparent diffusion coefficient in diffuse renal disease by diffusionweighted echo-planar MR imaging. J Magn Reson Imaging 1999; 9: 832-837.

16. Eldin S, Yadav S, Galal R, Abdelrahman W. DWI as a biomarker of renal function in children with CKD: what is the potential? Egyptian Journal of Radiology and Nuclear Medicine 2020; 171. DOI: https:// doi.org/10.1186/s43055-020-00277-0.

17. Haitian L, Zhangjian Z, Xiang Li, et al. Diffusion-weighted imaging for staging chronic kidney disease: a meta-analysis. Br J Radiol 2018; 91: 20170952.

18. Xu Y, Wang X, Jiang X. Relationship between the renal apparent diffusion coefficient and glomerular filtration rate: preliminary experience. J Magn Reson Imaging 2007; 26: 678-681.

19. Toya R, Naganawa S, Kawai H, et al. Correlation between estimated glomerular filtration rate and apparent diffusion coefficient values of kidneys. Magn Reson Med Sci 2010; 9: 59-64.

20. Emre T, Kilickesmez O, Buker A, Inal B, Dogan H, Ecder T. Renal function and diffusion-weighted imaging: a new method to diagnose kidney failure before losing half function. Radiol Med 2016; 121: 163-172.

21. Xue M, Ling Y, Jing R, et al. Non-invasive evaluation of renal structure and function of healthy individuals with multiparametric MRI effects of sex and age. Sci Rep 2019; 9: 10661.

22. Chandrana H, Vivian S, Hecht E, et al. Comparison of biexponential and monoexponential model of diffusion weighted imaging in evaluation of renal lesions. Invest Radiol 2011; 46: 285-291.

23. Xu X, Fang W, Ling H, et al. Diffusion-weighted MR imaging of kidneys in patients with chronic kidney disease: initial study. Eur Radiol 2010; 20: 978-983. 